\title{
Eine soziologische Unschärferelation
}

\section{Replik zum Aufsatz ,Is Free Speech in Danger on University Campus? Some Preliminary Evidence from a Most Likely Case“" von Matthias Revers und Richard Traunmüller}

\section{Lars Meier}

Eingegangen: 9. Februar 2021 / Angenommen: 16. Februar 2021 / Online publiziert: 26. April 2021 (C) Der/die Autor(en) 2021

Zusammenfassung Problematisiert wird, dass der Aufsatz von Revers und Traunmüller Erkenntnisinteresse und Positionalität der durchgeführten Forschung verschleiert. Eine Offenlegung wäre notwendig, um die Grundlagen der schwerwiegenden methodischen Probleme, der Fallauswahl und der unbelegten Behauptungen des Aufsatzes verstehen zu können. Im Widerspruch zu der falschen Annahme, dass Meinungsfreiheit grenzenlos sei und auch mit einer Freiheit einhergehe, andere zu diskriminieren, legt meine Replik die Notwendigkeit (siehe Grundgesetz und Gleichbehandlungsgesetz) dar, dass auch an den Universitäten Diskriminierungen aktiv vermieden werden müssen.

Schlüsselwörter Positionalität · Political correctness · Diskriminierungen · Kritik in Universitäten · Meinungsfreiheit 


\section{A Sociological Uncertainty Relation}

Reply to the Article "Is Free Speech in Danger on University Campus? Some Preliminary Evidence from a Most Likely Case" by Matthias Revers and Richard Traunmüller

Abstract It is problematic that the essay by Revers and Traunmüller obscures the epistemological interest and positionality of the research. Its disclosure would be necessary to understand the basis of the serious methodological problems, the case selection, and the unsubstantiated claims of the research article. Contrary to the false assumption that freedom of speech is without limits and also goes hand in hand with the freedom to discriminate against others, my reply sets out the need (see the Basic Law and the Equal Treatment Act) to actively avoid discrimination at universities.

Keywords Positionality of research - Political correctness - Discrimination · Criticism in universities $\cdot$ Freedom of speech

\section{Die Illusion wissenschaftlicher Neutralität}

Werner Heisenberg machte in seiner 1927 veröffentlichten Unschärferelation eine aufsehenerregende und nobelpreiswürdige Entdeckung. Der Physiker stellte fest, dass sich die Lage eines Elektrons mit seiner Betrachtung ändert und daher nicht exakt bestimmbar ist. Die Forschenden verändern den Aufenthaltsort des Elektrons mit dem Licht, das bei der Betrachtung auf das Elektron fällt und diesem einen Impuls gibt. Auch in den Sozialwissenschaften gibt es eine lange Tradition, die Forschung, ihre Konzipierung und Fragestellung, Planung, Durchführung und Auswertung nicht als unabhängig von den Forschenden und dem gesellschaftshistorischen Kontext zu verstehen, in dem diese stattfindet (beispielsweise verstanden als diskursiver Kontext bei Michel Foucault (1973) oder als Paradigma bei Thomas Kuhn (1967)). Meine Replik zu dem Aufsatz von Revers und Traunmüller ist nicht der passende Ort, um den Werturteilsstreit oder Positivismusstreit in der deutschen Soziologie umfassender nachzuvollziehen. Hingewiesen sei jedoch darauf, dass bereits Max Weber, der ansonsten eine Trennung von Forschung und wertender Betrachtung einforderte, eine vermeintliche Objektivität der Forschenden infrage stellte:

Es gibt keine schlechthin „objektive“ wissenschaftliche Analyse des Kulturlebens oder, - was vielleicht etwas Engeres, für unsern Zweck aber sicher nichts wesentlich anderes bedeutet, - der ,sozialen Erscheinungen“ unabhängig von speziellen und „einseitigen“ Gesichtspunkten, nach denen sie - ausdrücklich oder stillschweigend, bewußt oder unbewußt - als Forschungsobjekt ausgewählt, analysiert und darstellend gegliedert werden (Weber 1904, S. 45).

Dass die Forschenden selbst und ihre Positionalität in der Forschung eine Relevanz haben, wurde in vielen Arbeiten gezeigt und auch wissenschaftstheoretisch immer wieder hergeleitet und diskutiert. Dabei wird besonders darauf verwiesen, dass mit der Herleitung und Offenlegung des sozialen Standpunkts, des situierten Wissens (Haraway 1988; Harding 1991; Collins 2000) und dem Erkenntnisinteresse 
(Habermas 1968) der Forschenden eine bessere Annäherung an Objektivität möglich ist. Pierre Bourdieu zeigt, dass die Untersuchungsfelder der Soziologie grundsätzlich Felder von wissenschaftlichen und sozialen Kämpfen sind. Auch die Soziologinnen und Soziologen selbst haben mit ihrer spezifischen eigenen Kapitalausstattung und mit ihrer Position im Feld der Soziologie eine Position innerhalb dieser Kämpfe inne. Die Produktion von Wissen erfolgt demnach nicht unabhängig von den Forschenden, ihrem Blick (Clifford und Marcus 1986) und ihren Interessen. Daher schlussfolgert Bourdieu:

Gelingt es dem Soziologen (sic!), Wahrheit zu produzieren, dann nicht obwohl er (sic!) ein Interesse hat, diese Wahrheit zu produzieren, sondern weil er (sic!) dazu Interesse hat - was das genaue Gegenteil der etwas verdummten Rede von der „Neutralität“" ist (Bourdieu 1993, S. 23).

Demzufolge lässt sich eine objektivere Betrachtung der Sozialwelt nur erreichen, wenn die eigene Positionalität der Forschenden im sozialen Raum und die Position im sozialen Feld reflektiert und verdeckte Interessen und Absichten sichtbar gemacht werden (Bourdieu und Wacquant 1996). Die sozialwissenschaftliche Theoriebildung der letzten 100 Jahre macht deutlich, dass Wissen standortgebunden ist und sich hinter der Fassade einer angeblich völlig neutralen Wissenschaftlichkeit Vorurteile, Annahmen und Interessen verstecken. Werden die Positionen der Forschenden durch eine implizite Annahme einer von ihnen unabhängigen Forschung verschleiert, so birgt dies gravierende Probleme: Probleme, die im Beitrag von Revers und Traunmüller auf beeindruckende Weise deutlich werden.

\section{Verschleierte Positionalität im sozialen Feld der Universität}

In ihrem Beitrag, der erste empirische Belege für eine bedrohte Meinungsfreiheit an deutschen Universitäten zu liefern verspricht, begründen die Autoren ihre Fallauswahl - den Fachbereich Gesellschaftswissenschaften der Goethe-Universität Frankfurt - unter anderem mit der inhaltlichen Ausrichtung der Lehre. Die in dem Aufsatz behaupteten (alleinigen?) inhaltlichen Schwerpunkte der Lehre in den Feldern der Sprechakttheorie, Standpunkttheorie und Intersektionalität mache diese Universität und diesen Fachbereich zu einem ,most likely case“, die mögliche Einschränkung der Meinungsvielfalt zu messen. Nun mag man an diesem Punkt erwähnen, dass der Fachbereich Gesellschaftswissenschaften inhaltlich viel heterogener, multiparadigmatischer und vielfältiger ist, als dies in dem Beitrag dargestellt wird. Es bleibt unklar, warum die Autoren von einer solchen angeblichen Homogenität sprechen. Mit einem bloßen Blick auf die Internetseiten der Professuren und Arbeitsschwerpunkte oder in das Vorlesungsverzeichnis lässt sich diese Annahme widerlegen. Aber geht es eigentlich um den Fachbereich Gesellschaftswissenschaften oder um die Goethe-Universität als Ganzes? Dies wird nicht deutlich, denn mal wird in dem Beitrag die eine und mal die andere Institution adressiert. Wenn nur Studierende des Fachbereichs Gesellschaftswissenschaften befragt wurden, wie kann man dann die Ergebnisse auf die gesamte Universität übertragen? Aber diese schwerwiegenden methodischen Unklarheiten sind nicht das Hauptproblem des Beitrags. 
Vielmehr wird etwa an dieser mangelnden Begründung der Fallauswahl, die hauptsächlich auf der Basis unbelegter Behauptungen erfolgt, deutlich, dass eine Offenlegung der Interessen der Autoren zur Klarheit beitragen würde. Revers und Traunmüller schreiben, dass die Goethe-Universität (hier ist der Bezug die gesamte Universität) eine ,reputation as being a training ground for the political left“" hat. Wie und auf welcher Basis die Autoren zu dieser Einschätzung kommen, wird ebenso wenig belegt wie die Behauptung, ,the university is traditionally associated with the activist rather than the scientific impetus of the social sciences" (hier wird zuerst die Goethe-Universität als Ganzes und dann der Fachbereich Gesellschaftswissenschaften adressiert). Auch diese Aussage ist überraschend, da sich fragen lässt, ob es nicht gerade die in der Tradition der Frankfurter Schule stehende kritische Sozialforschung ist, die in den Sozialwissenschaften eine besondere, weltweit geachtete wissenschaftliche Bedeutung hat? Ebenso werden die wissenschaftlichen Erfolge und Reputationen der anderen am Fachbereich vertretenen Paradigmen hier beiläufig ohne irgendein nachvollziehbares datenbasiertes Argument entnannt. Auch die Ableitung einer allgemeingültigen Aussage, nach der es in Frankfurt (hier ist es unklar, ob es um die Universität, den Fachbereich oder die Stadt als Ganzes geht) eine idiosynkratrische Diskussionskultur geben soll, die mit einer einzigen Aussage eines interviewten Studierenden belegt wird, ist nicht nachvollziehbar.

Was also ist die Basis dieser unbelegten Behauptungen? Schritt für Schritt drängt sich der Eindruck auf, dass eine Reflektion der Positionalität und der Interessen der Autoren darauf eine Antwort geben könnte - sowohl hinsichtlich der Begründung der Fallauswahl wie auch im Hinblick auf die inhaltliche und darin auch politische Ausrichtung des Aufsatzes.

\section{Meinungsfreiheit als die Freiheit zu diskriminieren?}

Die Studie von Revers und Traunmüller fragt danach, ob es Belege gibt für ,,social pressure to conform to ,political correctness' (PC) and does this restrict free discussion on university campus?“. Damit schließt die inhaltliche Ausrichtung direkt an Narrative an, die, wie beispielsweise Texte von Thilo Sarrazin (2014), ,political correctness“ als eine einseitige Zensur und Einschränkung der Meinungsfreiheit darstellen (Niehr 2019). Die Basis dafür ist ein offensichtliches logisches Paradox. Während Positionen öffentlich formuliert werden, die einer sogenannten ,,political correctness" widersprechen, wird gleichzeitig eine Einschränkung der Meinungsfreiheit beklagt, nach der es nicht möglich sei, solche Positionen zu äußern. Diejenigen, die sich trotzdem äußern, werden dabei als mutige Vorbilder gerahmt, als diejenigen, die sich trotz angenommener Nachteile trauen zu sprechen. Auch bei der Annahme, dass an dem Fachbereich Gesellschaftswissenschaften der Goethe-Universität die Meinungsfreiheit - also ein Grundrecht - durch Studierende und Lehrende eingeschränkt wird, schwingt eine Bezugnahme auf dieses Narrativ mit. Die für die Forschung ausgewählten Fragen an die Studierenden haben den Charakter von Tabubrüchen und suggerieren, dass es mutig sei, solche der ,political correctness“ widersprechenden Fragen zu stellen. 
Im Ergebnis kommt der Beitrag zu dem Schluss, dass „taking offense is a common experience and that a sizable number of students are in favor of restricting free speech on university campus". Wie wurde aber in der Studie Meinungsfreiheit überhaupt getestet und verstanden? Gefragt wurden Studierende, ob Personen an der Universität sprechen oder lehren dürfen, die der Meinung sind, ,that there are biological differences in talents between men and women“, die gegen ,,all forms of immigration to this country“ sind, die glauben ,that Islam is incompatible with the Western way of life," oder die denken ,that homosexuality is immoral and dangerous." Getestet wurde damit nicht eine Einschränkung von Meinungsfreiheit im Allgemeinen. Sondern, man muss es präziser fassen, getestet wurde, ob es möglich ist, sich am Fachbereich Gesellschaftswissenschaften der Goethe-Universität Frankfurt rassistisch, antifeministisch und homophob äußern zu können, ohne Widerspruch befürchten zu müssen. Es wurde also gefragt, ob Studierende sich dafür aussprechen, dass solche Positionen nicht von anderen Studierenden oder Lehrenden geäußert werden.

Um eine Einschränkung von Meinungsfreiheit zu belegen, werden in dem Beitrag auch Erfahrungen von Studierenden angeführt, deren geäußerte Meinungen von anderen ,dissmissed or criticized“ wurden. Meinungsfreiheit wird damit auch gleichgesetzt mit einem Recht darauf, dass die eigene geäußerte Position nicht von anderen kritisiert oder abgelehnt wird. Ein solches Recht ist im Kern undemokratisch und widerspricht grundsätzlich dem diskursiven Ringen um Wahrheit an Universitäten, an denen es - anders als in Talkshows - um das Argumentieren auf Grundlage von wissenschaftlichen Studien und Ansätzen geht. Wenn man sich an eine Universität begibt, sollte es eine berechtigte Erwartung sein, dass dieses Ringen um Wahrheit mit Debatten und Kritik einhergeht.

Der Beitrag von Revers und Traunmüller unterliegt einem großen Irrtum. Denn dieser geht davon aus, dass das Recht auf Meinungsfreiheit quasi grenzenlos ist und auch das Recht umfasst, andere zu diskriminieren. Das Grundgesetz steckt hier in Artikel 3 einen engeren Rahmen und verbietet solche Diskriminierungen. Auch die Antidiskriminierungsstelle des Bundes und das allgemeine Gleichbehandlungsgesetz verfolgen das Ziel, Menschen zu unterstützen und zu schützen, die von solchen, auch sprachlichen Diskriminierungen betroffen sind. Viele Studierende erleiden auch an den Universitäten vielfältige Diskriminierungen, wie beispielsweise rassistische oder sexistische Abwertungen oder Benachteiligungen aufgrund der sozialen Herkunft (Antidiskriminierungsstelle des Bundes 2013). Warum sollten nun die Universitäten dazu im Widerspruch ein Klima schaffen, an denen solche Diskriminierungen sagbar sind? Es bleibt vor diesem Hintergrund unklar, was eigentlich daran problematisch ist, wenn Studierende von anderen dafür ,,reprimanded for using the word Rasse“ werden. Warum sollte die Nutzung eines Begriffs, der, gerade weil er diskriminierend ist, aus dem Grundgesetz gestrichen werden soll, unwidersprochen bleiben und warum sollten beispielsweise homosexuelle Menschen oder Menschen mit islamischem Glauben (sprachlichen) Diskriminierungen ausgesetzt sein? Wieso sollten Studierende und Lehrende gegen das Gleichbehandlungsgesetz handeln und nicht dazu beitragen Diskriminierungen in den Universitäten aktiv zu beseitigen und zu verhindern? 
Ohne Zweifel kann nicht angenommen werden, dass alle Studierenden zum Anfang ihres Studiums in der Lage sind, Diskriminierungsformen zu erkennen, ihre Wurzeln und Ausdrücke zu begreifen und das eigene, auch sprachliche Verhalten entsprechend zu reflektieren und anzupassen. Die Vermeidung von Diskriminierungen ist auch ein Teil des Lernprozesses im Rahmen von sozialwissenschaftlichen Studiengängen. Die Studierenden und Lehrenden an der Goethe-Universität sind dafür zu beglückwünschen, dass sie diese Lernprozesse forcieren und zwar auch, indem sie versuchen, solche diskriminierenden Äußerungen diskursiv und in Debatten zu kritisieren und zu vermeiden. Sie genau dafür zu kritisieren und ihnen für dieses Ringen eine Einschränkung der Meinungsfreiheit vorzuwerfen, ist der eigentliche Skandal.

Auch für die Forschung gilt, dass die eigene Positionalität der Forschenden offengelegt und produktiv für die Forschung genutzt werden muss. Der Impuls, der bei der Betrachtung von Elektronen erfolgt und keine exakte Bestimmung ihrer Lage ermöglicht, führte in der Physik zur Entwicklung neuer Atommodelle. In der Soziologie ist es die Positionalität der Forschenden, die eine solche Unschärfe produzieren und deren Reflektion bessere Einsichten in gesellschaftliche Zusammenhänge ermöglichen können.

Funding Open Access funding enabled and organized by Projekt DEAL.

Open Access Dieser Artikel wird unter der Creative Commons Namensnennung 4.0 International Lizenz veröffentlicht, welche die Nutzung, Vervielfältigung, Bearbeitung, Verbreitung und Wiedergabe in jeglichem Medium und Format erlaubt, sofern Sie den/die ursprünglichen Autor(en) und die Quelle ordnungsgemäß nennen, einen Link zur Creative Commons Lizenz beifügen und angeben, ob Änderungen vorgenommen wurden.

Die in diesem Artikel enthaltenen Bilder und sonstiges Drittmaterial unterliegen ebenfalls der genannten Creative Commons Lizenz, sofern sich aus der Abbildungslegende nichts anderes ergibt. Sofern das betreffende Material nicht unter der genannten Creative Commons Lizenz steht und die betreffende Handlung nicht nach gesetzlichen Vorschriften erlaubt ist, ist für die oben aufgeführten Weiterverwendungen des Materials die Einwilligung des jeweiligen Rechteinhabers einzuholen.

Weitere Details zur Lizenz entnehmen Sie bitte der Lizenzinformation auf http://creativecommons.org/ licenses/by/4.0/deed.de.

\section{Literatur}

Antidiskriminierungsstelle des Bundes. 2013: Diskriminierung im Bildungsbereich und im Arbeitsleben. Zweiter Gemeinsamer Bericht der Antidiskriminierungsstelle des Bundes und der in ihrem Zuständigkeitsbereich betroffenen Beauftragten der Bundesregierung und des Deutschen Bundestages. https://www.antidiskriminierungsstelle.de/SharedDocs/Downloads/DE/publikationen/BT_ Bericht/gemeinsamer_bericht_zweiter_2013.pdf?_blob=publicationFile\&v=7 (Zugegriffen: 02. März 2021).

Bourdieu, Pierre. 1993. Soziologische Fragen. Frankfurt a. M.: Suhrkamp.

Bourdieu, Pierre, und Loïc Wacquant. 1996. Reflexive Anthropologie. Frankfurt a. M.: Suhrkamp.

Clifford, James, und Marcus George (Hrsg.). 1986. Writing culture: The poetics and politics of ethnography. Berkeley: University of California Press.

Collins, Patricia Hill. 2000. Black feminist thought. Knowledge, consciousness, and the politics of empowerment. London: Routledge.

Foucault, Michel. 1973. Archäologie des Wissens. Frankfurt a. M.: Suhrkamp.

Habermas, Jürgen. 1968. Erkenntnis und Interesse. Frankfurt a. M.: Suhrkamp. 
Haraway, Donna.1988. Situated Knowledges. The Science Question in Feminism and the Privilege of Partial Perspective. Feminist Studies 14(3):575-599.

Harding, Sandra. 1991. Feministische Wissenschaftstheorie - Zum Verhältnis von Wissenschaft und sozialem Geschlecht. Hamburg: Argument Verlag.

Kuhn, Thomas S. 1967. Die Struktur wissenschaftlicher Revolutionen. Frankfurt a. M.:Suhrkamp.

Niehr, Thomas. 2019. Sprache - Macht - Gewalt oder: wie man die Grenzen des Sagbaren verschiebt. Sprachreport 35(3):1-7.

Sarrazin, Thilo. 2014. Der neue Tugendterror. Über die Grenzen der Meinungsfreiheit in Deutschland. München: DVA.

Weber, Max. 1904. Die „Objektivität“ sozialwissenschaftlicher und sozialpolitischer Erkenntnis; Archiv für Sozialwissenschaft und Sozialpolitik 19(1):22-87.

Lars Meier Dr., Professor für Soziologie mit dem Schwerpunkt Soziale Ungleichheit, Institut für Soziologie, Goethe-Universität Frankfurt am Main. Forschungsgebiete: Soziale Ungleichheiten, sozialräumliche Transformationen, Ethnographie. Wichtigste Veröffentlichungen u.a. in Journals wie Cultural Geographies, Cultural Studies, International Sociology, Sociological Review. Bücher u. a.: Working Class Experiences of Social Inequalities in (Post-)Industrial Landscapes - Feelings of Class. New York 2021; Theoretische Positionen der Stadtsoziologie. Weinheim 2018 (mit S. Steets und L. Frers); Migrant Professionals in the City - Local Encounters, Identities, and Inequalities. New York 2015 (als Hrsg.). 